Delft University of Technology

\title{
Designing a $100[\mathrm{aF} / \mathrm{nm}]$ capacitive transducer
}

Middelburg, L.M.; Mansouri, B. El; Poelma, R.H.; Van Zeijl, H.W.; Wei, J.; Zhang, G.Q.; Van Driel, W.D.

DOI

10.1109/EuroSimE.2018.8369954

Publication date

2018

Document Version

Final published version

Published in

2018 19th International Conference on Thermal, Mechanical and Multi-Physics Simulation and Experiments in Microelectronics and Microsystems, EuroSimE 2018

\section{Citation (APA)}

Middelburg, L. M., Mansouri, B. E., Poelma, R. H., Van Zeijl, H. W., Wei, J., Zhang, G. Q., \& Van Driel, W. D. (2018). Designing a 100 [aF/nm] capacitive transducer. In 2018 19th International Conference on Thermal, Mechanical and Multi-Physics Simulation and Experiments in Microelectronics and Microsystems, EuroSimE 2018 (pp. 1-3). IEEE . https://doi.org/10.1109/EuroSimE.2018.8369954

\section{Important note}

To cite this publication, please use the final published version (if applicable).

Please check the document version above.

\section{Copyright}

Other than for strictly personal use, it is not permitted to download, forward or distribute the text or part of it, without the consent of the author(s) and/or copyright holder(s), unless the work is under an open content license such as Creative Commons.

Takedown policy

Please contact us and provide details if you believe this document breaches copyrights.

We will remove access to the work immediately and investigate your claim. 
Green Open Access added to TU Delft Institutional Repository

'You share, we take care!' - Taverne project

https://www.openaccess.nl/en/you-share-we-take-care

Otherwise as indicated in the copyright section: the publisher is the copyright holder of this work and the author uses the Dutch legislation to make this work public. 


\title{
Designing a $100[\mathrm{aF} / \mathrm{nm}]$ capacitive transducer
}

\author{
$\underline{\text { L.M. Middelburg}}{ }^{1}$, B. El Mansouri ${ }^{1}$, R.H. Poelma ${ }^{1}$, H.W. van Zeijl ${ }^{1}$, J. Wei ${ }^{1}$, G.Q. Zhang ${ }^{1}$, W.D. van Driel ${ }^{1,2}$ \\ ${ }^{1}$ Delft University of Technology, Dept. of Microelectronics, Mekelweg 4, 2628CD, Delft, The Netherlands \\ ${ }^{2}$ Philips Lighting, HTC Eindhoven, The Netherlands
}

\begin{abstract}
The mechanical part of inertial sensors can be designed to have a large mechanical sensitivity, but also requires the transduction mechanism which translates this displacement. The overall system resolution in mechanical inertial sensors is dictated by the noise contribution of each stage and the magnitude of each sensitivity, see also Figure 1. Maximizing the capacitive sensitivity, results in suppression of noise in the electronics domain. This work focuses on the design and realization of a mechanical to electrical transduction using a capacitive readout scheme. Design considerations and measures are taken to maximize the latter are considered and illustrated using FEM simulations. A capacitive transducer showing a sensitivity of 100 [aF/nm] was designed and realized, by exploiting the large displacement behavior of the inertial sensor which was considered.
\end{abstract}

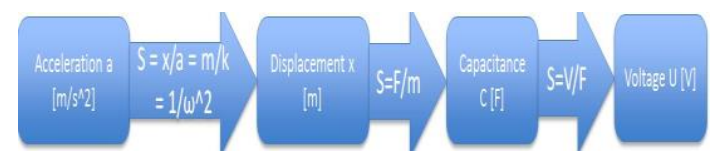

Figure 1: System overview of the inertial sensor considered.

\section{Introduction}

Various applications require acceleration measurements using MEMS inertial sensors, for example the airbag application. However, some applications require extremely high sensitivities such as lithography machines where $\mathrm{nm}$ resolution is required or other applications where measuring small vibrations is a requirement [1][2]. Thus, designing inertial sensors for acceleration values in the nano- $g$ range is a requirement for various applications. Within this context this work focusses on the design and modelling of an interdigitated capacitive transducer which is optimized to have a maximum capacitive response of $2[\mathrm{aF} / \mathrm{nm}]$ within the boundaries of the available technology. A vertically aligned inertial sensor is considered which uses a nonlinear movement to introduce high sensitivity for small vibrations. The transduction of the mechanical to electrical domain is done using capacitive interdigitated structures where the high sensitivity was obtained by maximizing the number of fingers within a $10 \mathrm{~mm}$ distance and by exploiting the self-aligning and metastability of the mechanical part of the inertial sensor. The device is planarly designed to operate vertically in line with the normal component of the gravitational force of the earth. This results in a relative large displacement where the proof mass shifts by approximately $2 \mathrm{~mm}$. The large displacement is guided using sliders to align the two comb shaped electrodes on each side of the capacitor to form an interdigitated (IDT) electrode configuration for the capacitive read-out. Figure 2 illustrates the horizontal view of the device showing the IDT structures and a guider. The system can be considered meta-stable due to the shifting action over a large displacement. The use of the self-aligning IDT structure allowed the gap size of the comb structure to be independent on the lateral resolution limit introduced by photo-lithography and DRIE etch processes.

The device was realized by employing bulk silicon micro-machining, where Deep Reactive Ion Etching (DRIE) was used to etch through a $300 \mu \mathrm{m}$ substrate. In this process, the lateral resolution using this technology is limited to around $40 \mu \mathrm{m}$ which in turn limits the number of IDT fingers, hence a lower capacitive change. Thanks to the meta-stable shifting of the IDT fingers, a much smaller gap size between both electrodes was obtained which resulted in the dramatic increase of the capacitive sensitivity.

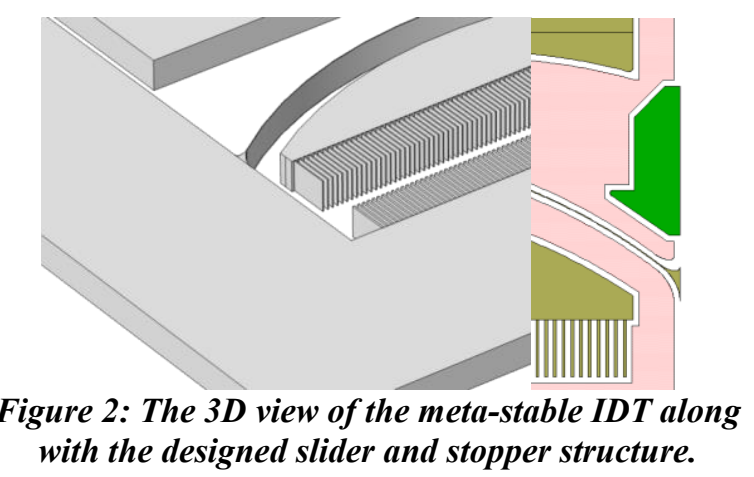

To verify the desired behavior, solid mechanics and electrostatic simulations have been performed using COMSOL Multiphysics ${ }^{\circledR}$. A two-step simulation was performed where first the behavior of the accelerometer was done after which the IDT capacitor was modeled.

Since the inertial sensor is based on nonlinear behavior one needs to take some measures into account to ensure convergence of the simulation to a valid solution. For the solid mechanics simulations, multiple manners can be taken to ensure convergence in the case of large displacement. The first way is based on documentation [3] and uses an average operator on the displacement of the springs in the resonator system. This average displacement is subsequently used as an input parameter by the model to determine the force. This is done by using a global equation, in which the difference between this 
average operator and an explicit value for the desired displacement is put to zero. In this way basically an additional equation and an additional unknown is added to the model. It is important not to over- or under- constrain the problem when using these global equations. The advantage of this way of solving, is that we can now specify a displacement rather than an force. By doing so COMSOL is applying the force on the proof mass implicitly. To make sure that this mechanism is not diverging to unrealistic values, an additional stop condition was used. A second method is to use a prescribed displacement in the physics section of the simulation. When doing so, an auxiliary sweep is required to gradually converge to the desired point of deflection.

\section{Results}

The electric field distribution is included in Figure 3 in the form of an electric potential distribution. The electric potential increases from zero (ground) to 5 [V], which equals the amplitude of the square wave used later by the impedance read-out ASIC. Further it can be noticed that the top electrode is grounded, which has reasons related to system design considerations. The capacitive response of the designed IDT capacitive structure is included in Figure 4. Although the presence of fringing E-field effects on either side of the finger array, the capacitance as a function of a (prescribed) displacement still exhibits a highly linear behavior. This type of structure was first introduced by Tang et al. [4] to overcome the nonlinear behavior of parallel plate capacitors. The high degree of linearity can be explained by the large number of fingers. The non-linear effect of the fringing E-field on either side of the electrode array is suppressed by the large contribution to the total capacitance of the normal components of the field between the parallel 'fingers'. Additionally it can be noted that the aspect ratio of the structure is high, the width of each finger equals only $20 \mu \mathrm{m}$, whereas the thickness of the structure equals wafer thickness of 300 $\mu \mathrm{m}$, a property which is also responsible for the large suppression of the fringing fields on either side of each individual finger.

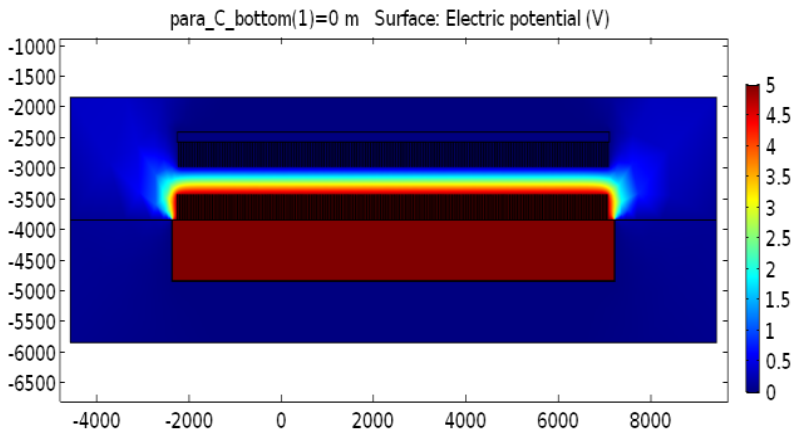

Figure 3: The potential of the interdigitated structure.

As for the damping, the lateral comb drive structure helps to reduce damping where couette flow occurs for lateral motion, which is much less dissipative compared to the squeeze film damping. The damping should be low since the frequency of interest is $1 \mathrm{~Hz}$ or lower. This is because the frequency of operation is low, the fluid inside the gaps has enough time to displace allowing for less dissipation but also preventing the air to act like a spring that constantly stores and releases energy at higher frequencies [5]. Additionally, the damping can be further reduced by using vacuum packaging. It can be seen from Figure 4 that the capacitance first shows a parallel plate behavior (1/d) before the IDT electrodes are shifted into each other and thus a small capacitive change per nm displacement. After the large displacement of approximately $2 \mathrm{~mm}$ the IDT structured capacitor is formed, yielding a significantly higher sensitivity. Taking the first order derivative of the capacitance versus displacement characteristic yields a sensitivity $\left(\mathrm{S}_{\mathrm{cap}}\right)$ of $100[\mathrm{aF} / \mathrm{nm}]$ in the displacement region of interest.

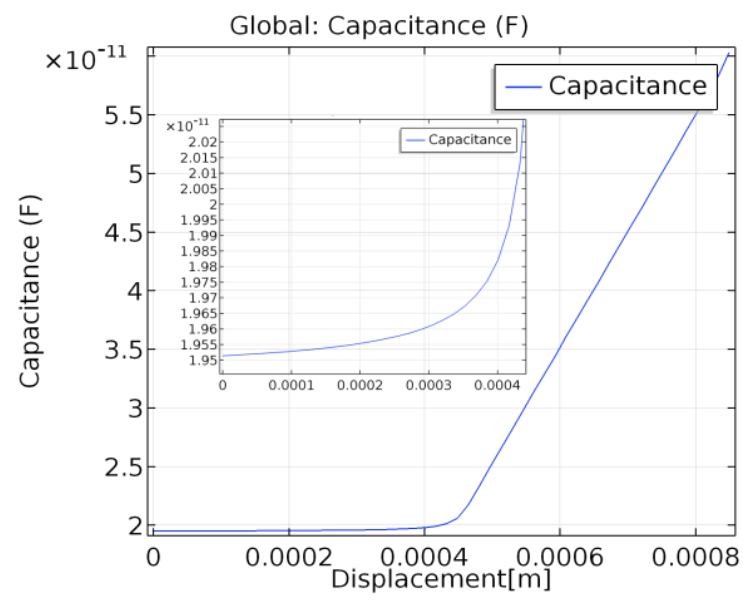

Figure 4: The simulation result of the linearly increasing capacitance as function of the deflection, after the two comb structures of the meta-stable system form a capacitor. The transition from the parallel plate behaviour to the IDT behaviour is clearly visible.

\section{Realization}

The design of the IDT structure was transferred from COMSOL into a mask design. By designing the mask to be modular, two different widths for the fingers of the bottom electrode array were obtained. This was done as a redundant measure to ensure process feasibility.

The MEMS device was fabricated using standard IC technology where bulk micromachining was used to etch the structures in a $300 \mu \mathrm{m}$ substrate. Figure 5 illustrates the final device.
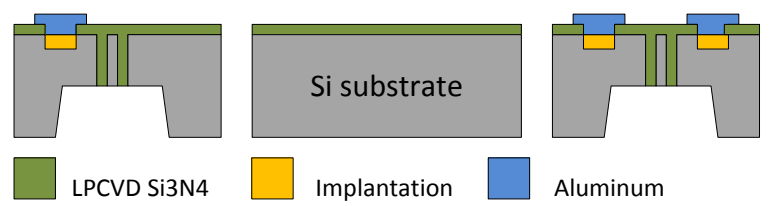

Figure 5: Illustration of realized device.

Since the substrate is also used as an electrode, first electrically isolated islands were formed into the substrate using bulk micromachining and subsequently an 
implantation step was performed to make ohmic contacts. The etched trenches were then filled using LPCVD Si3N4 for insulation and adhesion of the islands to the main substrate. Figure 6 shows the resulting trench, it can be noted that a high AR profile is obtained.

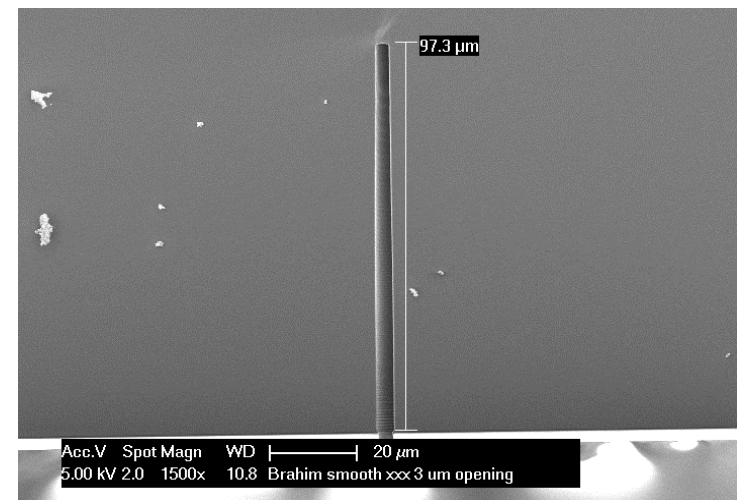

Figure 6: Etched isolation trench into the Si substrate.

After this an Al metallization step was performed for interconnect and patterned for later connecting the device. Subsequently the substrate was coated with PECVD SiO2 as a hard mask on both sides for etching through from the front side while etching from the bottom side to reach the insulation material for electrical isolation. At the backside also a $3 \mu \mathrm{m}$ Al layer was deposited prior to etching to make sure no helium flow leakage is detected during the DRIE etch step in case the PECVD SiO2 landing layer at the backside cracks. The devices where then diced and cleaned. The last steps were the $\mathrm{Al}$ and oxide layers removal using ICP etching and vapor etch, respectively. Figure 7 shows the resulting IDT structure including also a guider for the proof mass.

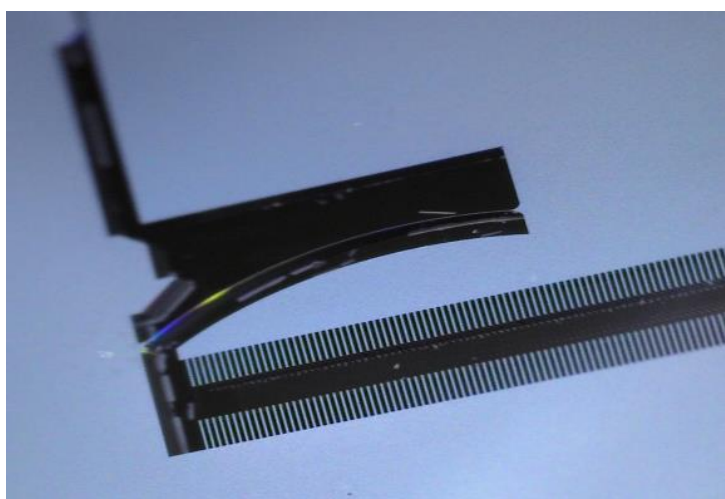

Figure 7: A microscope picture of the realized capacitive transducer.

\section{Conclusions}

The large displacement behavior of a non-linear massspring system was exploited in the design of a high sensitivity capacitive transducer. By using a self-aligning IDT structure, the gap size of the comb structure is not dependent on the lateral resolution limit introduced by photo-lithography and DRIE etch processes.

COMSOL FEM simulations were used to verify the capacitive response and to investigate possible non-linear behavior caused by non-ideal stray effects on the boundaries and edges of the structure. These are however suppressed by the large number of fingers and the high aspect ratio of the device, concluding from the simulation result.

The device has been fabricated within the accelerometer structure. The aligning property was verified.

\section{Acknowledgments}

This work was supported by the European project "IoSense: Flexible FE/BE Sensor Pilot Line for the Internet of Everything". This project has received funding from the Electronic Component Systems for European Leadership Joint Undertaking under grant agreement No 692480. This Joint Undertaking receives support from the European Union's Horizon 2020 research and innovation programme and Germany, Saxony, Austria, Belgium, Netherlands, Slovakia, Spain.

\section{References}

[1] Middlemiss, R. P., A. Samarelli, D. J. Paul, J. Hough, S. Rowan and G. D. Hammond (2016). "Measurement of the Earth tides with a MEMS gravimeter." Nature 531(7596): 614-617.

[2] Li, Z., W. J. Wu, P. P. Zheng, J. Q. Liu, J. Fan and L. C. Tu (2016). "Novel Capacitive Sensing System Design of a Microelectromechanical Systems Accelerometer for Gravity Measurement Applications." Micromachines 7(9): 167.

[3] COMSOL Multiphysics, accessed 2017. "Loaded Spring - Using Global Equations to Satisfy Constraints" https://www.comsol.com/model/download $/ 383611 /$ model s.mph.loaded spring.pdf

[4] Tang, W. C., Nguyen, T. C. H., \& Howe, R. T. (1989). Laterally driven polysilicon resonant microstructures.

Sensors and actuators, 20(1-2), 25-32.

[5] Hajhashemi, M. S., Rasouli, A., \& Bahreyni, B. (2014). Performance optimization of high order RF microresonators in the presence of squeezed film damping. Sensors and Actuators A: Physical, 216, 266276. 